Portland State University

PDXScholar

6-15-2017

\title{
Ethanol-Nicotine Combinations Induces C-Fos Expression within Stress and Reward-Related Circuitry
}

Jacob J. Schoen

Portland State University

Follow this and additional works at: https://pdxscholar.library.pdx.edu/honorstheses

Let us know how access to this document benefits you.

\section{Recommended Citation}

Schoen, Jacob J., "Ethanol-Nicotine Combinations Induces C-Fos Expression within Stress and RewardRelated Circuitry" (2017). University Honors Theses. Paper 491.

https://doi.org/10.15760/honors.494

This Thesis is brought to you for free and open access. It has been accepted for inclusion in University Honors Theses by an authorized administrator of PDXScholar. Please contact us if we can make this document more accessible: pdxscholar@pdx.edu. 


\section{ETHANOL-NICOTINE COMBINATIONS \\ INDUCES C-FOS \\ EXPRESSION WITHIN \\ STRESS AND REWARD- \\ RELATED CIRCUITRY}

by

Jacob J. Schoen

An undergraduate honors thesis submitted in partial fulfillment of the requirements for the degree of

Bachelor of Arts

in

Psychology

Under the Advisement of

Matthew M. Ford Ph.D

Portland State University 
Research completed at

Division of Neuroscience, Oregon National Primate Research Center and Department of

Behavioral Neuroscience, Oregon Health \& Science University, Portland, OR, USA 


\section{List of Abbreviations}

$\begin{array}{ll}\text { ACTH } & \text { Adrenocorticotropic Hormone } \\ \text { cpEW } & \text { Centrally Projecting Edinger-Westphal Nucleus } \\ \text { CDC } & \text { Center for Disease Control } \\ \text { CNS } & \text { Central Nervous System } \\ \text { CRH } & \text { Corticotropin-Releasing Hormone } \\ \text { DA } & \text { Dopamine } \\ \text { GABA } & \text { Gamma-Aminobutyric Acid } \\ \text { HPA } & \text { Hypothalamic-Pituitary-Adrenal } \\ \text { IACUC } & \text { Institutional Animal Care and Use Committee } \\ \text { IEG } & \text { Immediate Early Gene } \\ \text { LS } & \text { Lateral Septum } \\ \text { nAChRs } & \text { Nicotinic Acetylcholine Receptors } \\ \text { NuAcc } & \text { Nucleus Accumbens } \\ \text { ROIs } & \text { Regions of Interest } \\ \text { VTA } & \text { Ventral Tegmental Area }\end{array}$





\section{Table of Contents}

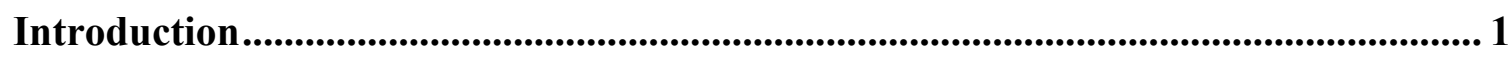

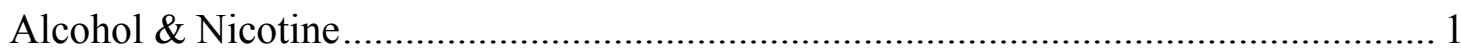

Animal Model of Co-Abuse ........................................................................... 3

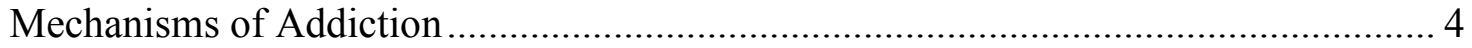

Neural Circuitry Underlying Alcohol and Nicotine Effects ................................... 7

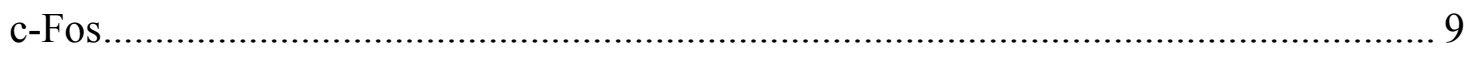

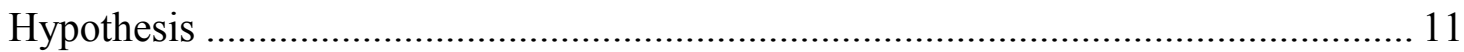

Methods........................................................................................................................................ 13

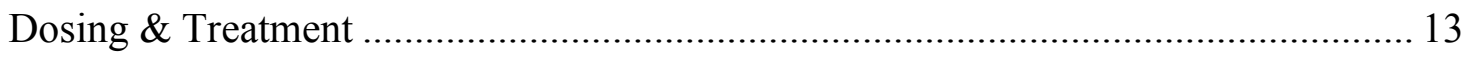

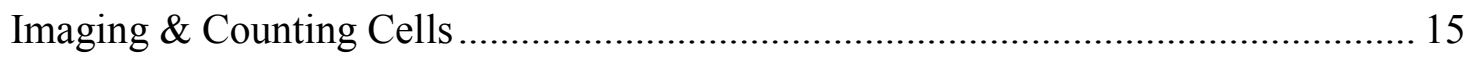

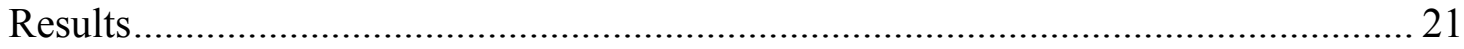

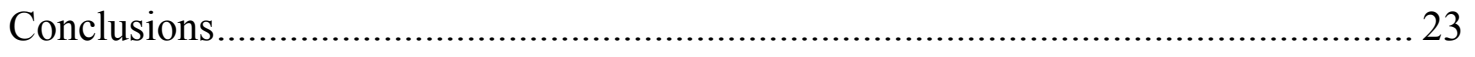

Bibliography ....................................................................................................................... 25 


\section{List of Figures}

Figure 1: Light Microscope with Mounted Camera ................................................... 16

Figure 2: Region of Interest (ROI) Image J ........................................................ 17

Figure 3: Subtracted Background Black and White Image J ..................................... 18

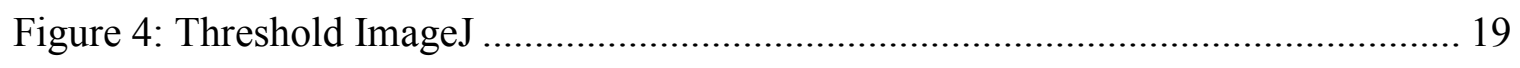

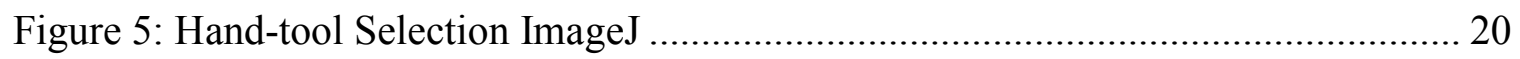




\section{Abstract}

The use and abuse of alcohol and nicotine are intimately related, with co-use of alcohol among adult cigarette smokers higher $(>65 \%)$ than non-smokers $(>45 \%)$, and smoking rates among alcoholics above $75 \%$. Smoking is associated with an accelerated progression to alcohol dependence, suggesting that nicotine and alcohol act in a synergistic manner to promote coabuse. While recent work has begun to identify discrete 'neuronal ensembles' within stress and reward circuitry components that underlie nicotine's contribution to escalations in alcohol selfadministration, the neurobiological mechanisms facilitating alcohol-nicotine interactions following chronic drug exposure remain understudied. The goal of the current work was to identify a network of brain regions in mice that are activated differentially following chronic intermittent exposure to alcohol versus alcohol plus nicotine combinations via c-Fos immunoreactivity. Male C57BL/6J mice were systemically treated with either alcohol (1.5 g/kg) or a combination of alcohol plus nicotine $(0.4,0.8$, or $1.2 \mathrm{mg} / \mathrm{kg}$ base $)$ on a double-alternation schedule (drug, drug, saline, saline) for a 13-month period. Mice were euthanized 90-min following the final treatment, whole brains were removed and $40-\mu \mathrm{m}$ coronal sections were evaluated for c-Fos immunoreactivity using established laboratory procedures. Co-administration of 0.4 and $0.8 \mathrm{mg} / \mathrm{kg}$ nicotine dose-dependently enhanced c-Fos immunoreactivity in the ventral tegmental area (VTA), centrally projecting Edinger-Westphal nucleus (cpEW), and the lateral septum (LS) when compared to alcohol only treated mice. In contrast, co-administration of 1.2 $\mathrm{mg} / \mathrm{kg}$ resulted in a $33 \%$ decline in c-Fos labeled cells within the VTA, no change in LS activation, and comparable activation to $0.4 \mathrm{mg} / \mathrm{kg}$ nicotine in the $\mathrm{cpEW}$. In summary, chronic intermittent nicotine exposure exacerbates the c-Fos response to alcohol in a dose-dependent and brain region-selective fashion. The nearly uniform, maximal response observed following 0.8 $\mathrm{mg} / \mathrm{kg}$ nicotine across stress- and reward-associated brain regions is consistent with the ability of this dose to accelerate the progression towards excessive alcohol self-administration and to potentiate the discriminative stimulus effects of alcohol in rodents. 
-Dedicated to my Dad - 


\section{Introduction}

\section{Alcohol \& Nicotine}

The use of Alcohol and Tobacco products has become a major public health concern in recent years. Nicotine specifically is the primary component in tobacco products that leads to drug dependence and reward. Alcohol and nicotine have pharmacological and neurological synergistic effects, resulting in various health problems such as cancers, vascular diseases, and gastric ulcers (Hurley, Taylor, \& Tizabi, 2012). Widespread legalization and abundant availability plays a role in these most commonly abused substances, but genetic, rewarding, and analgesic properties of alcohol and nicotine also add to massive levels of use among the public. Alcohol and nicotine are often used simultaneously, especially among alcoholics, where smoking rates are estimated to be about twice as much as the general population in both adolescents and adults (Falk, Yi, \& Hiller-Sturmhöfel, 2006). This is a growing concern because traditional addiction research has only set out to study the effects of alcohol and nicotine separate from one another. This approach to addiction research does not accurately represent the way in which alcoholics and smokers consume these substances. In turn, the use of alcohol among adult cigarette smokers is relatively high $(65.2 \%)$ compared to non-smokers $(48.7 \%)(C D C, 2013)$. Acute nicotine administration has also been shown to increase alcohol self-administration in non-dependent smokers, suggesting that nicotine dose can effect alcohol's interaction with the brain early in the process of addiction 
(Barrett, Tichauer, Leyton, \& Pihl, 2006). The current research aims to address the question of how chronic, long term exposure, to alcohol and nicotine may influence the way these drugs are taken over time by creating a novel set of 'neuronal ensembles' that utilize previously associated mechanisms of addiction. With these questions in mind, knowing how various dosing levels of nicotine react with a consistent dose of alcohol can aid in finding the neurological mechanisms underlying co-abuse. Nicotine seems to prime the reward and stress system for alcohol, resulting in a greater amount of alcohol administration, and produces long lasting changes to signaling pathways (Doyon et al., 2013). It is currently unclear as to how co-morbidity arises from the interaction of these two drugs, but further exploration of nicotine addiction can help reveal many aspects of drug dependence, especially in adolescents and people with mental illness, who are at higher risk for co-morbidity (Dani \& Harris, 2005). As a way to incorporate running hypotheses within the field of addiction research, the involvement of stress hormones and associated stress-hormone releasing structures within the brain are included in the analysis of 'neuronal ensembles', but it is still under investigation as to how exactly these stress pathways are influencing the neurology and behaviors of addiction. Stress-related hormones have been found to have lasting changes in dopamine and GABA neurotransmission, which are active components of the reward pathway (Walker et al., 2012). GABA acts primarily as an inhibitory neurotransmitter, with activation typically causing a decrease in neuronal excitability in the central nervous system (CNS), with notable building evidence to a possible link between the GABA type A $\left(\mathrm{GABA}_{\mathrm{A}}\right)$ receptor and the genetic component of alcoholism (Davies, 2003). Alcohol acts partially on the $\mathrm{GABA}_{\mathrm{A}}$ receptors to enhance receptor activity and increasing the amount of 
GABA available. Dopamine, on the other hand, is associated with all drugs of abuse and is commonly associated by researchers with wanting, learning, and reward. The relationship between dopamine and other neurotransmitters, especially GABA, is vital to understanding the way in which these networks of addiction and reward operate. The receptors where nicotine act in the brain are thought to be primarily nicotinic acetylcholine receptors (nAChRs), and are involved in both the mediation of alcohol and the cause of behavioral changes in nicotine (Bito-Onon, Simms, Chatterjee, Holgate, \& Bartlett, 2011). nAChRs are found in many areas in the brain, including those related to reward and stress (Ventral Tegmental Area, Substantia Nigra, Striatum, Hypothalamus). Exploration of the basic mechanisms involved in co-abuse are needed before it can be fully understood as to how these neurotransmitters, receptors, and pathways are interacting with alcohol and nicotine. By studying the overlapping actions of alcohol and nicotine, valuable information about the co-abuse of many drugs of addiction can be revealed. These efforts lay down the groundwork for further research not only in the field of addiction, but for any study of neurological interactions and mechanisms. This approach can effectively narrow the direction of how treatments are used and made accessible for public use.

\section{Animal Model of Co-Abuse}

As a way of addressing issues in studying co-abuse, neurological activity must be examined to reveal specific areas of the brain that are most active when alcohol and nicotine are present. Although substance abuse is a human concern, using a suitable 
animal model can provide valuable behavioral and neurological data on alcohol-nicotine interactions. It is unlikely that an exact representation of the complexity of human addiction can be reached using the animal model alone, but specific aspects can be revealed such as drug self administration, tolerance, sensitivity, and withdrawal (Balogh, Owens, Butt, Wehner, \& Collins, 2002). Procedures of self-administration have shown that rats will continually consume significant amounts of both alcohol and nicotine when made available, with levels of nicotine self-administration similar to rates of nicotine alone (Lê et al., 2010). This research supports the important relationship between the two drugs, and the need to use animal models to ask basic questions about how are interacting. Animal models can be used as a way to address questions that both fully utilize the particular model and maximize results for comparison and analysis.

\section{Mechanisms of Addiction}

Abused drugs act on a series of neurological mechanisms within the brain, specifically the reward system. All individuals use the reward system every day for many aspects of life including eating, drinking, sex, and social engagement. The reinforcement of behaviors that are beneficial for survival are pleasurable to us because of the function of the reward system. We continue to partake in these activities because the neurological pathways are strengthened after each interaction, establishing a memory of reward. Drugs of addiction utilize the same reward pathways to alter an individual's natural drive towards drug seeking behaviors. Because the brain is communicating via electrical and chemical signaling, drugs can change the way in which this signaling process happens, by either strengthening or weakening the communication between neurons. The chemical 
signal used by neurons are called neurotransmitters, and when released, bind to receptors that activate a cascade of cellular activity. Dopamine (DA) is the key neurotransmitter involved in the reward system, but GABA, glutamate, and serotonin among others are also involved. DAergic neurons project from the VTA and midbrain structures to limbic and cortical areas, including the Nucleus Accumbens and the Prefrontal Cortex when activated by pleasurable stimuli. When dopamine is released in the form of a chemical message, it either binds to receptors or is taken back up through transporters reuse. Drugs of addiction, such as alcohol and nicotine, indirectly excite the neurons that produce dopamine in the VTA to generate a greater amount of dopamine release within the synapse. The repeated pulse of dopamine receptor activation will de-sensitize the reward system over time, which decreases the normal dopamine response to healthy and everyday rewarding activities. When drugs are used often over a long period of time, as with chronic drug users, the brain begins to adapt to these changes. A patterned response is then established, which is referred to as neuroadaptation. The response from the brain is a developed tolerance, where the same doses of the drug no longer produce the same effect and larger doses are needed to get the desired gratification. Drugs of addiction will quickly rise to a top priority for users with a compromised reward system, because these drugs give greater pleasure than other activities. Over time, the dopamine receptors will become overstimulated and stop responding to the previous dose of the drug, which eventually leads to permanent damages to the way the brain functions in everyday activity.

The stress system adds an additional feature to the way drugs are delivering the experience of reward and pleasure. The hypothalamic-pituitary-adrenal (HPA) axis is 
activated when our body naturally reacts to stress. The hypothalamus is a small structure that regulates the release of hormones from the pituitary gland. The release of hormones from the pituitary gland travel down the bloodstream to the kidneys and interact with the adrenal glands. The regulation of the stress response begins at the hypothalamus, where a hormone called corticotropin-releasing hormone $(\mathrm{CRH})$ is released. $\mathrm{CRH}$ signals to the pituitary gland to secrete adrenocorticotropic hormone (ACTH) into the bloodstream, which travels down to the adrenal glands and signals the release of different steroid hormones depending on the site of activation. A class of hormones released from the outer (cortical) layer of the adrenal glands are called glucocorticoids, specifically cortisol, and they play and important role in stress, which increase blood sugar, suppress the immune system, and aid metabolism. ACTH also acts on the adrenal medulla (center of the adrenal gland) to trigger the release of epinephrine and norepinephrine, thereby increasing heart rate and alertness. Elevated cortisol levels in the bloodstream are sensed by glucocorticoid receptors in the hypothalamus that initiate a negative feedback response to counteract the stress response. This regulation via negative feedback allows for the regulation of cortisol to maintained at appropriate levels. Glucocorticoid receptors are also found in the midbrain, striatum, and cortical areas important for reward. It has been suggested that a nicotine and alcohol combination alters stress hormones in the early stages of drug intake and prime the system for negative emotional states, which are described as withdrawal (George F. Koob et al., 2014). This further supports the idea that having a nicotine addiction (i.e. smoking cigarettes) can make a person more susceptible to drinking high amounts of alcohol. 


\section{Neural Circuitry Underlying Alcohol and Nicotine Effects}

Alcohol (or ethanol specifically) is a dynamic drug that reacts with several brain functions. Generally, alcohol is known as a depressant that inhibits brain activity in the CNS. Alcohol interacts with GABA-A receptors to gate the amount of negatively charged ions (i.e., chloride) that are let into the cell. Chloride acts to inhibit the excitability of the neuron by causing hyperpolarization. For drinkers of alcohol, results of the interaction with GABA-A receptors produce calming and anxiolytic effects at low doses, but negative effects impair judgement and disrupt motor functions at higher doses. NMDA (glutamate) receptors, which are associated with the excitability of the neuron, are inhibited by alcohol by blocking the gating of sodium $(\mathrm{Na}+)$ and calcium $(\mathrm{Ca}+2)$ into cells. Nicotine is a psychostimulant that acts on acetylcholine receptors (nAChRs) to depolarize the neuron and increase excitability. Within the sympathetic nervous system nicotine increases blood pressure, heart rate, and the release of norepinephrine. Cigarette smokers experience effects of calmness, alertness, relaxation, but negative symptoms of craving, irritability, and impatience.

When these drugs are taken together, both biological and behavioral effects are altered. The complex phenotypes that are observed in addicted individuals vary depending on the drug(s) of choice, resulting in different neurological changes within these circuits. Although neuronal activation following alcohol or nicotine can be observed separately, the combination of the two drugs likely alters the way these pathways would function during single drug use (for instance, synergism or additivity) or involves activation of new pathways in the brain. To begin to understand how alcohol 
and nicotine are interacting we must gain a perspective on previous addiction studies, findings, and definitions. A commonly accepted addiction model describes three progressive stages of the addiction that involve binge, withdrawal, and craving (G. F. Koob, 1997). These stages have been associated with discrete circuits that incorporate specific neuronal structures that describe how theories of addiction can be applied to a variety of drugs. Ventral tegmental area (VTA) and ventral striatum are associated with binge, amygdala with withdrawal, and prefrontal cortex, basolateral amygdala, hippocampus, and insula with craving (G F Koob \& Volkow, 2010). Response to chronic exposure tends to be more restricted within certain brain regions in the case of alcohol. On the other hand, consistent response from both chronic and acute treatments of alcohol are seen from lateral septum, edinger-westphal nucleus, and paraventricular nucleus of the hypothalamus (Vilpoux, Warnault, Pierrefiche, Daoust, \& Naassila, 2009). The use of mecamylamine (nACh-R antagonist) or other ligands that bind to receptors activated by alcohol or nicotine may allow for a clear view of what types of activity are happening with or without this substance. Nicotine speeds up the process of alcohol drinking in dependent rats, recruiting a number of neuronal structures including VTA and dorsomedial prefrontal cortex, blocking nicotinic receptors with mecamylamine (noncompetitive antagonist of nAChRs), effectively blocking behavioral and neuronal effects (Leão et al., 2015). Drugs that replace the effects of alcohol or nicotine can help to determine what each substance is acting on receptors and interacting with each other. The influence of nAChR antagonist mecamylamine has been examined within operant selfadministration in C57BL/6J mice, and found a suppression in dose-dependent alcohol intake and change in locomotor activity (Ford et al., 2009). Although more experiments 
using drug substitutions still need to take place, the previous findings suggest that a map of the way in which nAChRs and neuronal activity function with the combination of alcohol and nicotine would be of valuable importance for addiction research.

\section{c-Fos}

C-Fos is the cellular counterpart to a viral gene isolated in 1982 from FinkelBiskis-Jinkus osteogenic sarcoma virus, and is defined as a type of immediate-early gene (IEG) that rapidly responds to intracellular signaling (Sng, Taniura, \& Yoneda, 2004). IEGs have been an important focus of the field of addiction research for over 20 years because administering drugs of addiction (chronic or acute) changes IEG expression, which can be used to measure the processes of addiction in a way that identifies druginduced neuroplastic changes (Kalivas et al., 2006). Positive c-Fos activation has been identified using immunocytochemistry to reveal effects of nicotine on brain regions related to stress, suggesting they could be mediating the effects of nicotine on the central nervous system (CNS) (Matta, Valentine, \& Sharp, 1997). C-Fos can be stained in the brain by using an antibody to reveal the regions activated prior to mice being euthanized. We would expect the traditional reward and stress regions to be active, but c-Fos can reveal new areas of activation and significant suppression or expression differences in varied doses. 


\section{Research Question}

How does chronic intermittent administration of alcohol and alcohol-nicotine combinations change the amount of brain activation in the male C57BL/6J mouse brain?

\section{Hypothesis}

It was hypothesized that the combined effects of alcohol and nicotine would produce brain activation in stress and reward related areas, with the combined alcoholnicotine dosing (fixed alcohol dose plus low, medium or high dose nicotine) showing a difference in activation compared to the saline or alcohol only control groups. 


\section{Methods}

C57BL/6J (B6) mice were acquired from the Jackson Laboratory-West (Sacramento, CA, USA) for the drug discrimination (behavioral) portion of this study (beyond the scope of this thesis; data not shown). All the mice were male, and double housed in standard laboratory cages. Lights were turned on from $6 \mathrm{am}-6 \mathrm{pm}$ each day to regulate sleep cycles. The mice were given food, and maintained at $90 \%$ of their freefeeding body weights. Water was freely available. The Institutional Animal Care and Use Committee (IACUC) approved all procedures with the animals in accordance with state and federal guidelines. IACUC members are made up of qualified and experienced experts that oversee animal programs, facilities, and procedures (Ford, McCracken, Davis, Ryabinin, \& Grant, 2012).

\section{Dosing \& Treatment}

Mice groups were administered a fixed alcohol dose $(1.5 \mathrm{~g} / \mathrm{kg})$ or one of three combinations of alcohol + nicotine. Treatment groups were as follows: $1.5 \mathrm{~g} / \mathrm{kg}$ alcohol (1.5E) alone, $1.5 \mathrm{~g} / \mathrm{kg}$ alcohol $+0.4 \mathrm{mg} / \mathrm{kg}$ nicotine $(1.5 \mathrm{E}+0.4 \mathrm{~N}), 1.5 \mathrm{~g} / \mathrm{kg}+0.8 \mathrm{mg} / \mathrm{kg}$ nicotine $(1.5 \mathrm{E}+0.8 \mathrm{~N})$, and $1.5 \mathrm{~g} / \mathrm{kg}$ alcohol $+1.2 \mathrm{mg} / \mathrm{kg}$ nicotine $(1.5 \mathrm{E}+1.2 \mathrm{~N})$. Fixed ratio schedule (FR) was used as a form of operant conditioning that provided reinforcement after a specified number of responses. Once the mice were trained, the mice were divided into separate groups and given an intraperitoneal (i.p.) injection of either the saline, alcohol, or nicotine-alcohol combination (Ford et al., 2012). A double- 
alternation schedule of drug administration was used where saline (i.e., non-drug) was given two days in a row and then alcohol or both drugs for two days (saline, saline, drug, drug). Each group of mice were required to meet criteria for drug discrimination for three days prior to and including the final testing session, the last requirement that the mice needed to demonstrate criteria on the test session that day in order to be euthanized (see Ford et al., 2012 for additional details). To avoid disruptions in home cage environments, both mice (housed in pairs) were required to meet criteria on the same day. Each group $(n=12)$ was divided into sub-groups $(n=6)$ that were balanced with a four day mean according to total responses from both levers (activity measure), initial FR accuracy (\% appropriate responding), and body weight (training sessions only; test sessions not included). The time of day, handling, and transportation were all taken in to consideration during the procedure for final testing, as to not disrupt normal occurrences in the home cage. Individual mice were injected with treatment dose according to sub-group and placed into the operant chamber for 10-min pretreatment. Following pretreatment, the house light turning on and the test session began for the standard 15 min sessions. The mice were then returned to their home cage and fed their daily food diet, and remained in the procedural room for another 55 min before being transported to a separate procedural room where they were placed in a $\mathrm{CO}_{2}$ chamber. At 90 min total after initial treatment injection mice were euthanized (Ford et al., 2012). Normally, a perfusion of paraformaldehyde (PFA) is used to preserve the tissue and organs of the mouse, but in this particular procedure PFA was not used because it can prevent the primary antibody (c-Fos) from binding properly. The whole brain was isolated in a time sensitive manner (about $12 \mathrm{ml}$ of fixative tissue) and placed into a cold a $2 \%$ paraformaldehyde buffer. The 
brains can then be cryoprotected and stored for up to year or more. When brains are ready to be sliced they are placed on a fixative mount, covered with a thin layer of Optimal Cutting Medium (OCT), and frozen before being cut into slices 30-40 microns thick. After the slices are placed into net wells they are stained with the primary antibody (cFos), washed, and dried before coverslipping with cryoseal (Ryabinin, Criado, Henriksen, Bloom, \& Wilson, 1997). The final product of this procedure allows for the stained brain slices to be viewed under a microscope for comparison and analysis.

\section{Imaging \& Counting Cells}

An initial global examination of the mouse brain slides was taken using a light microscope and a mouse brain atlas. This allowed for an unbiased look at c-Fos activation throughout different sections of the brain. Certain areas with staining that could be seen easily with the naked eye stuck out immediately, but it was still difficult to get an accurate count of cell activation (ranging from 10-2000 cells) along with having a way to to detect the variations in size, shape, and clarity of the stained cells. The traditional method for counting cells, done by hand, would not provide a reliable count of the cells that were stained on each slide and because some sections contained thousands of cells, we needed an accurate way to measure and analyze these cell populations for statistical comparison. To best examine each brain slice, digitalized each of the slices using a light microscope and a camera (mounted on top). 


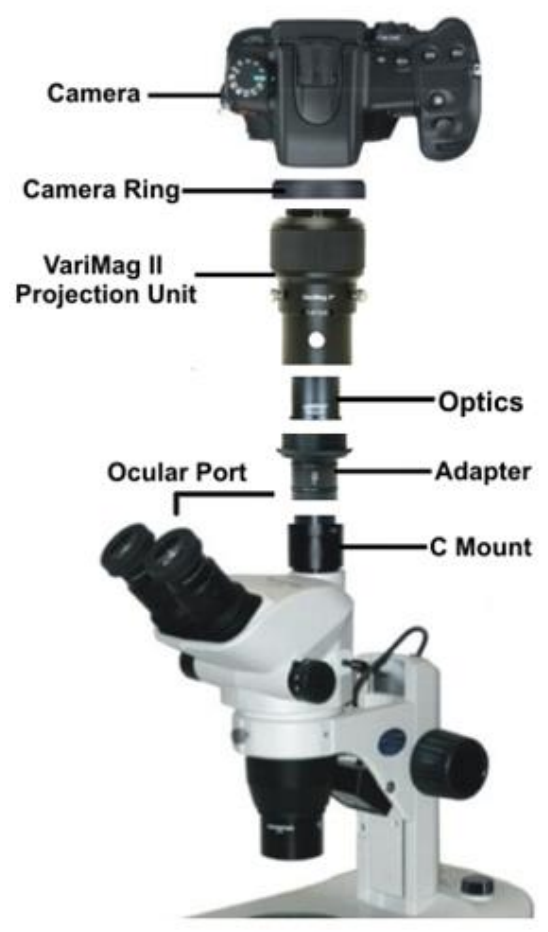

Figure 1: Light Microscope with Mounted Camera

The camera itself was hooked up to a computer monitor for live viewing of the microscope slides. This provided an accurate way to see how the image would turn out before taking the shot. The camera settings were kept consistent to preserve uniformity between images.

This proved to aid in the clarity of the staining along with providing the ability to use additional analysis programs. With the help of Andrey Ryabinin's lab at OHSU Marquam Hill we were able to get a series of images from the following brain regions including: Lateral Septum (LS), Edinger-Westphal Nucleus (EW), Substantia Nigra (SN)(Bi-Lateral), Nucleus Accumbens (NuAcc)(Bi-Lateral), and Ventral Tegmental Area (VTA)(Bi-Lateral) (OHSU, n.d.). Beginning with each brain region, settings on the light microscope and mounted camera were kept at consistent settings, with a background image of blank light taken at the start of each session. The background image would later be subtracted from the brain image to reduce any excess light and allow for a clearer view 
of the staining. The regions of interest (ROIs) were imaged and organized by mouse number and slide. To keep the images consistent across regions the light microscope would be rented out for 4-8 hours at a time to capture the entire selection. For the analysis, ImageJ was used, an open-source java-based processing program developed at the National Institutes of Health (NIH) to provide the architecture to create a procedure for counting c-Fos cells (ImageJ, n.d.) Each image was properly rotated and examined for any obstructive light, dirt, or user interference.

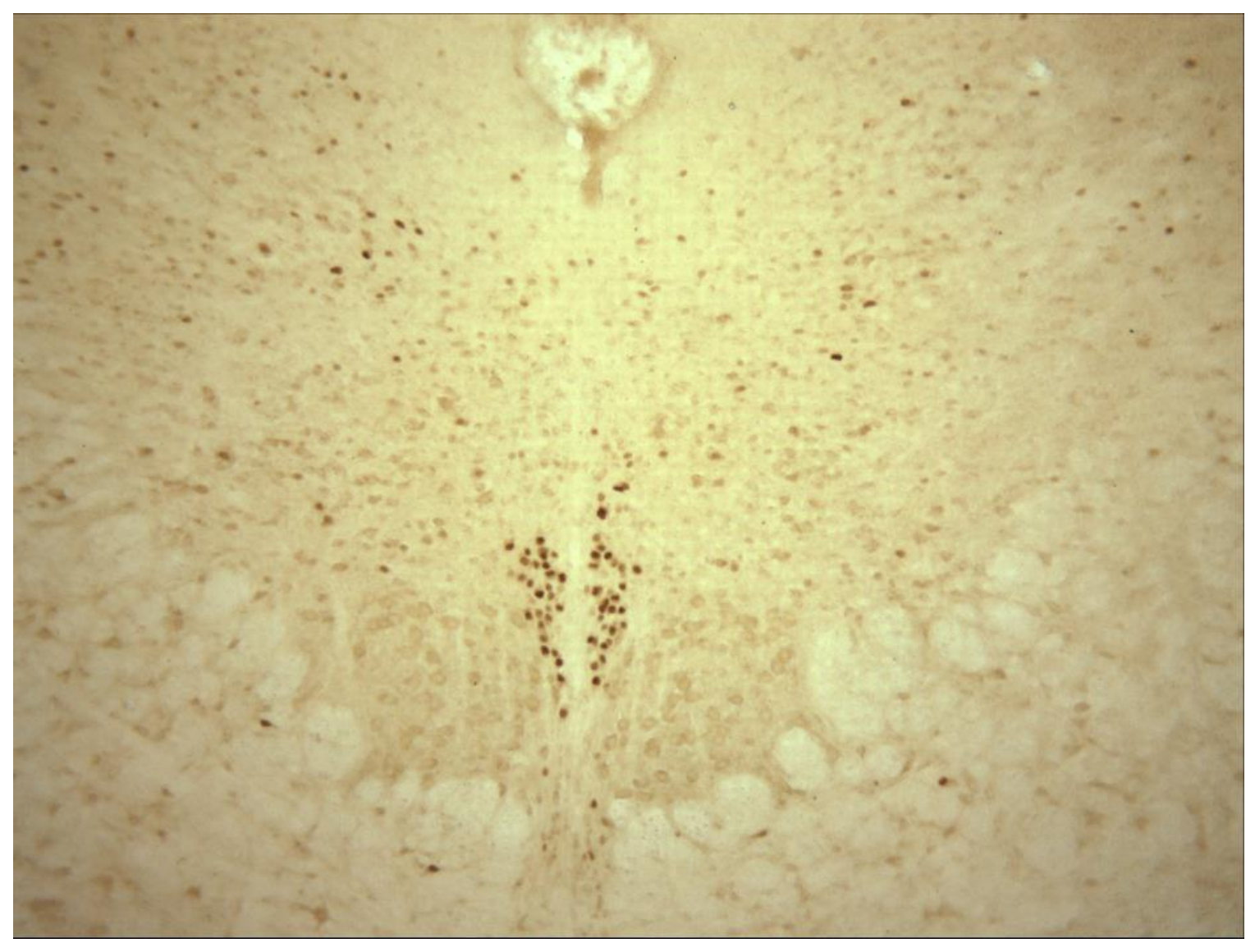

Figure 2: Region of Interest (ROI) ImageJ

The region above (EW) is an un-edited zoomed-in captured image (10x) of a mouse brain slice. Because each slice image needed to be consistent, none of the slides were edited for contrast or acuity. This required a great deal of attention to the way in which each image was captured. 
The background image and the ROI image was opened in ImageJ, and the background was subtracted. A new black and white ROI image was then generated to allow for a noise free analysis of the stained cells.

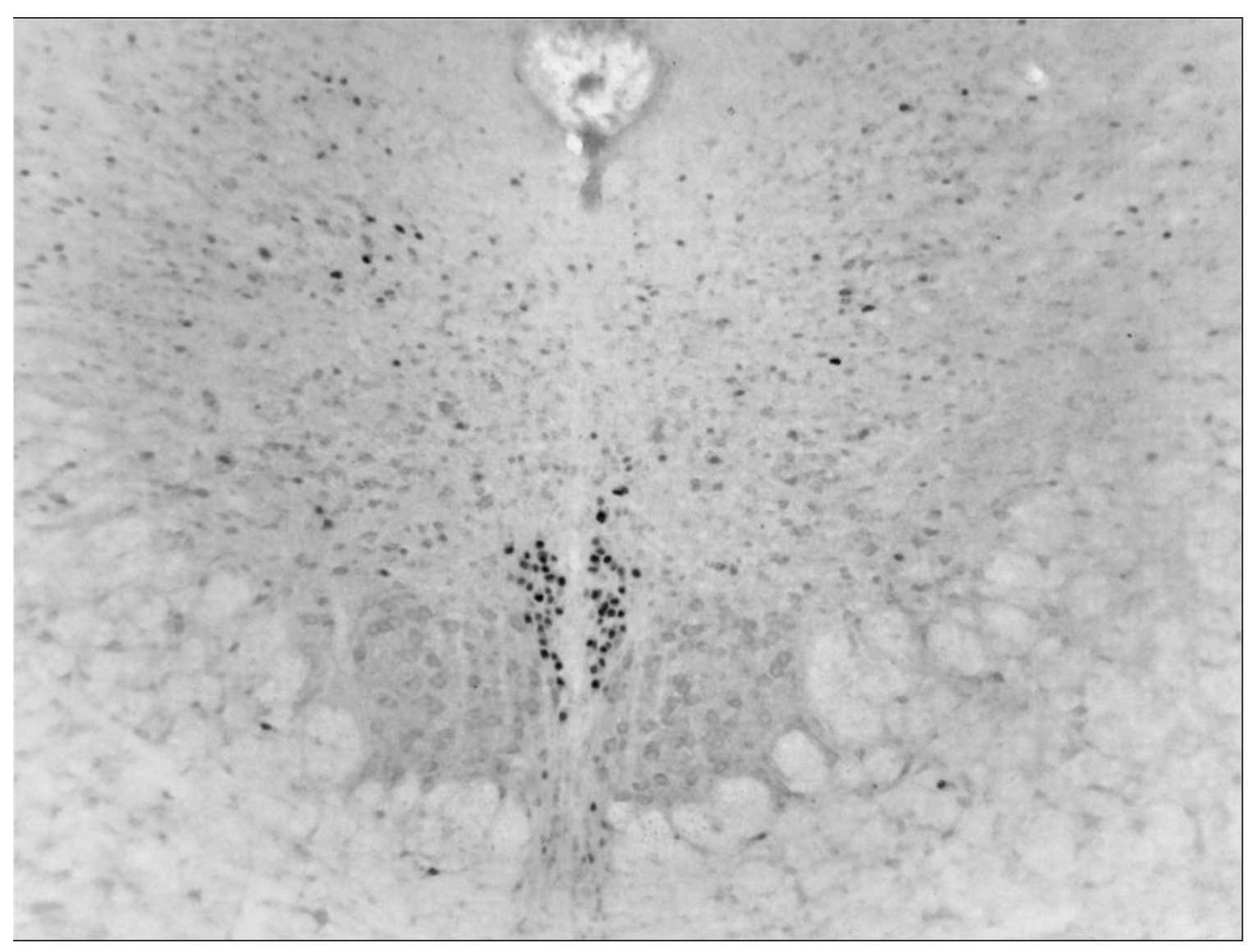

Figure 3: Subtracted Background Black and White ImageJ

The purpose of subtracting the background allows for a clear view of the tissue only without the noise of the ambient light from the microscope, The black and white image allows for a greater contrast to help reveal the stained cell bodies.

The threshold tool within ImageJ was adjusted to fill up of the cell bodies, and a standard threshold criteria was established (10-110 pixels^2). 


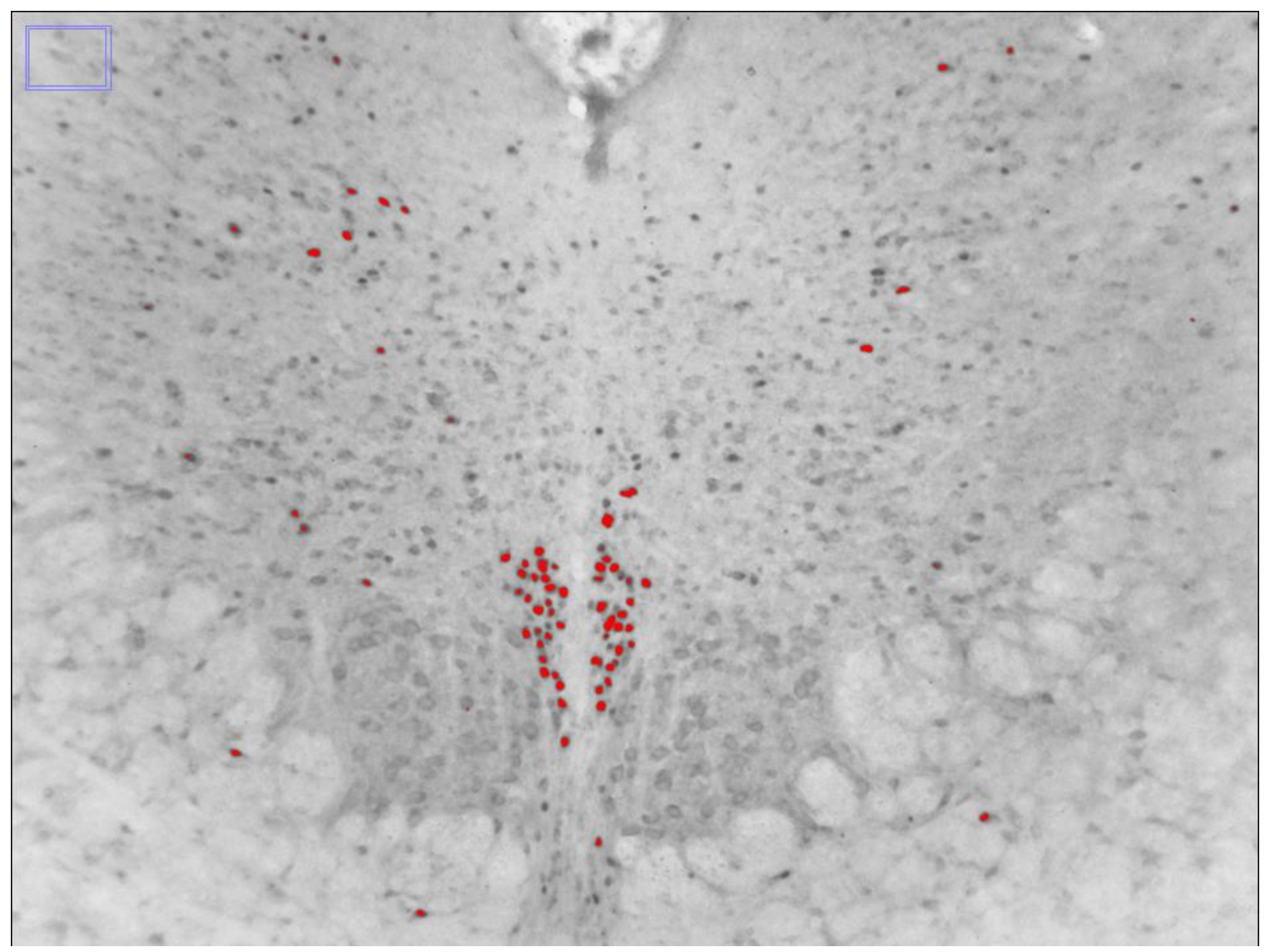

Figure 4: Threshold ImageJ

The threshold acts as a mask on top of the subtracted background image. The red selections can be adjusted until only stained cell bodies are highlighted. During the analysis portion the perimeters for each cell size is set to exclude any particles too large or small to be a cell.

A free hand selection method and a mouse brain atlas was used to select the ROI within the image to adjust for the size variance between brain slices and regions. After selecting the ROI, everything outside the selection was cleared and an analysis of the cell particles were run using ImageJ tools. 


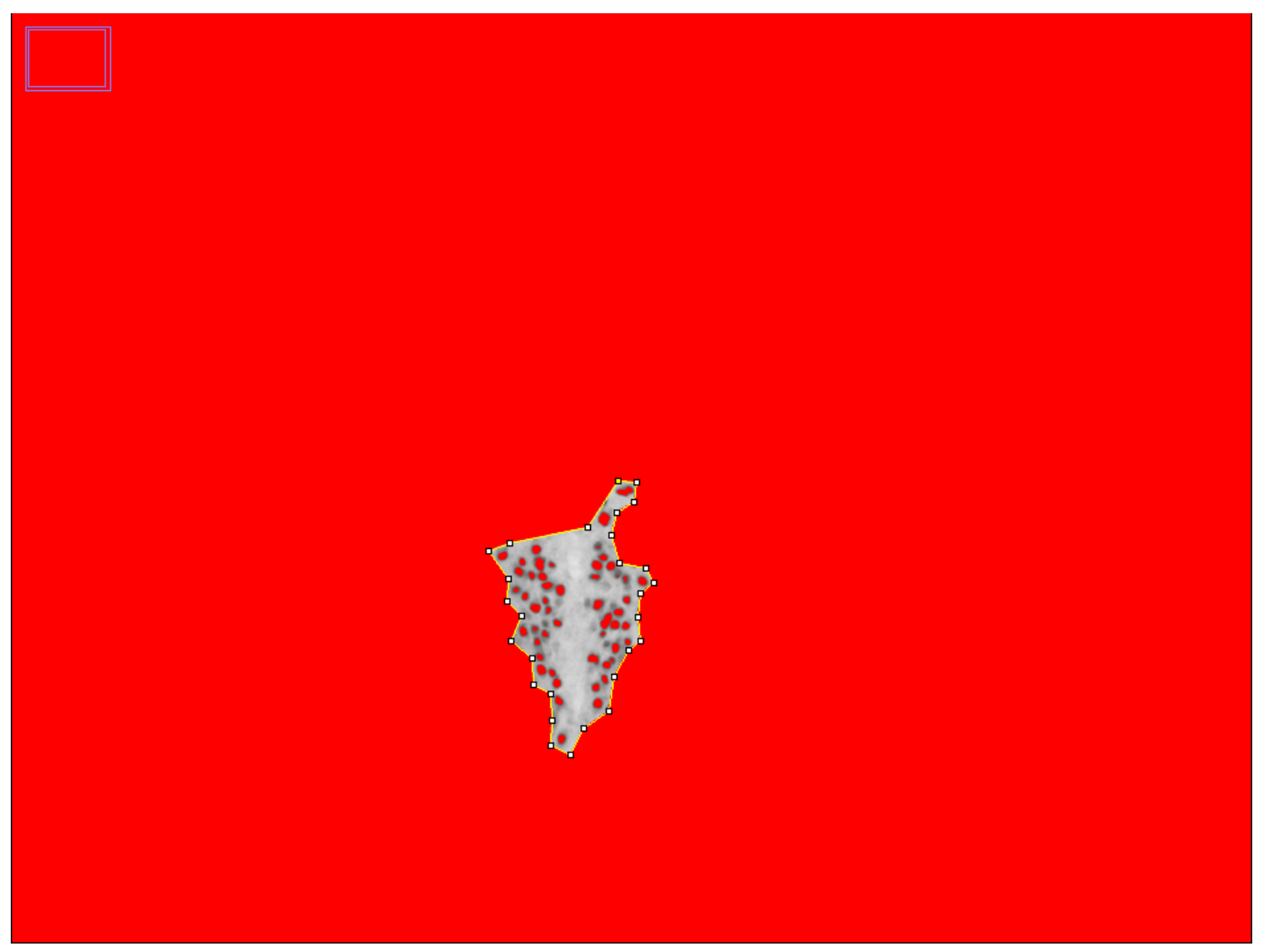

Figure 5: Hand-tool Selection ImageJ

The mouse brain atlas (Paxinos and Franklin's $4^{\text {th }}$ Edition) was used to identify the brain region stained was consistent with the ROI before making a hand drawn selection around the area. Everything outside the selected region is then cleared to make sure outside particles are not picked up in the cell count analysis.

A summary of the cell counts, total region area, and average cell size was generated for each slice (3-6 slices per mouse). Statistical data was analyzed using two way ANOVA to compare $0.4 \mathrm{~N}, 0.8 \mathrm{~N}$, and $1.2 \mathrm{~N}$ doses to the ethanol only treated group and comparisons between individual groups. Averages were obtained by combining all slice counts from each mouse ( $\mathrm{n}=5-6$ mice per group). 
Results

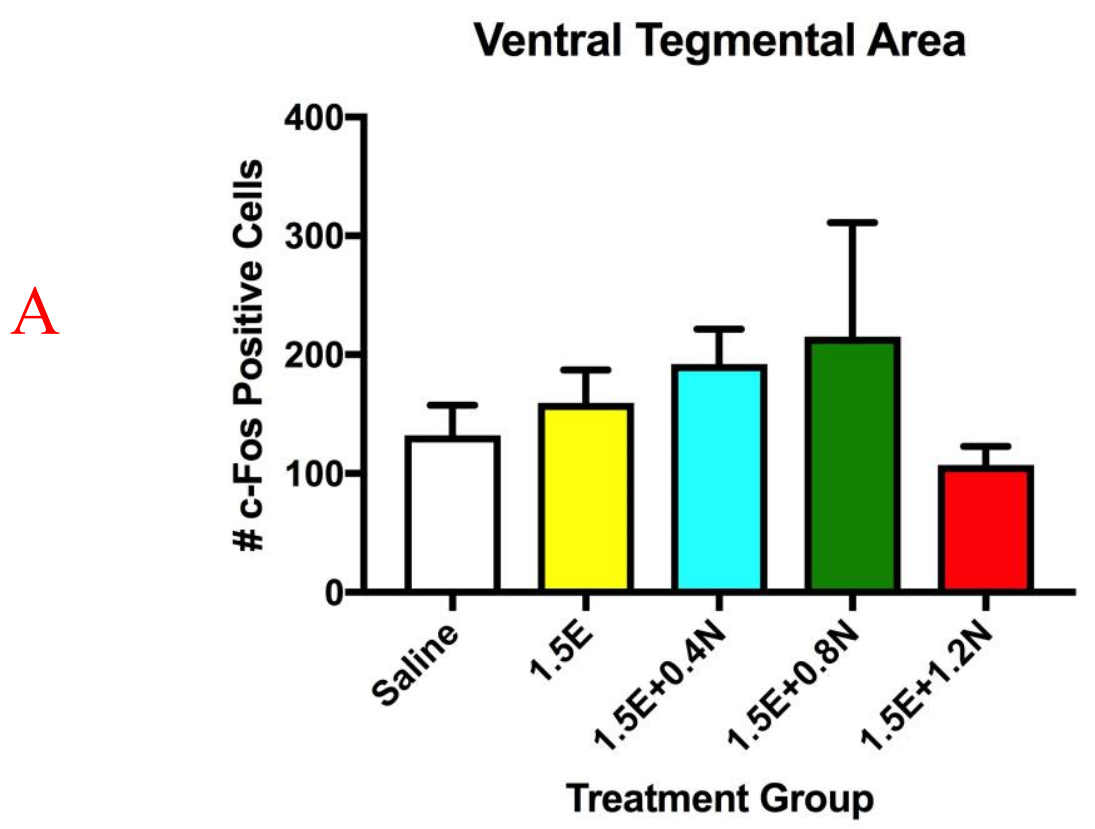

Edinger-Westphal Nucleus

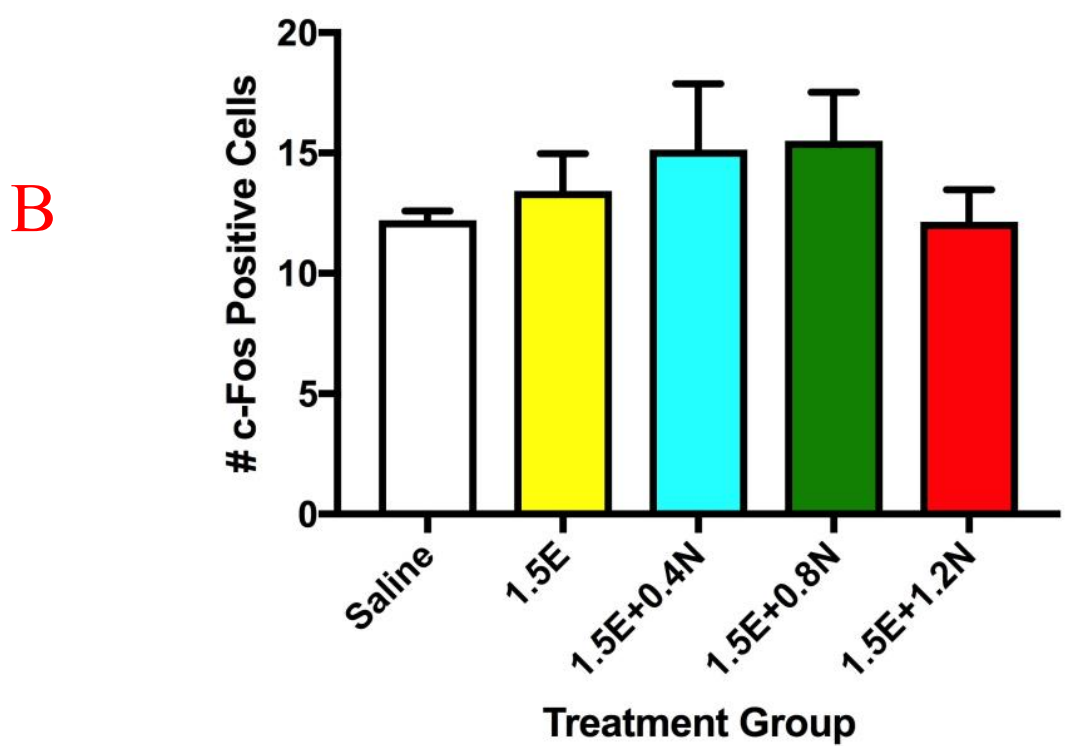


Lateral Septum

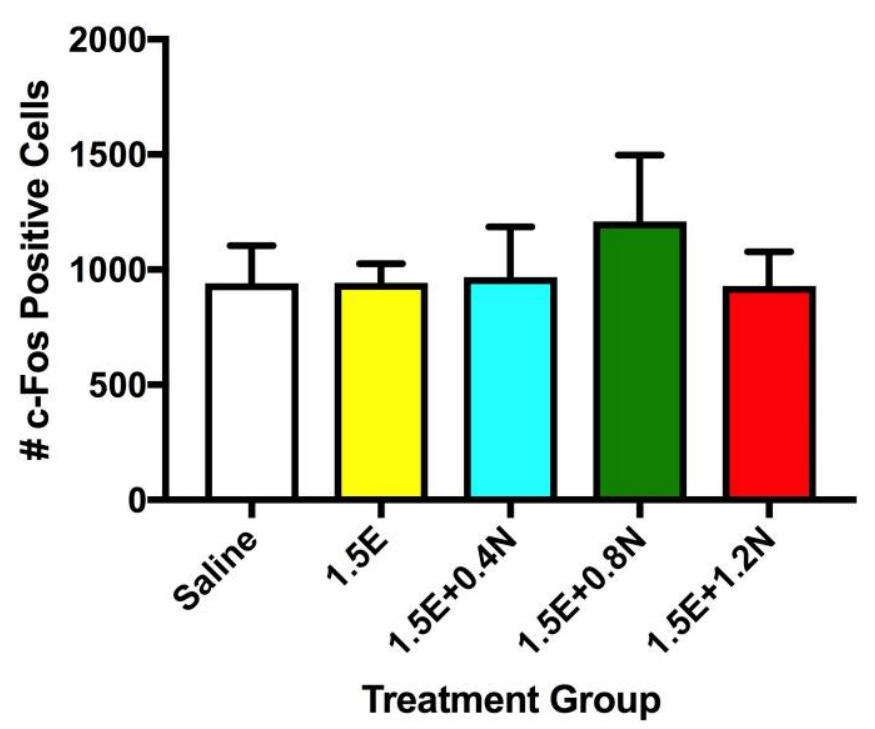

Nucleus Accumbens

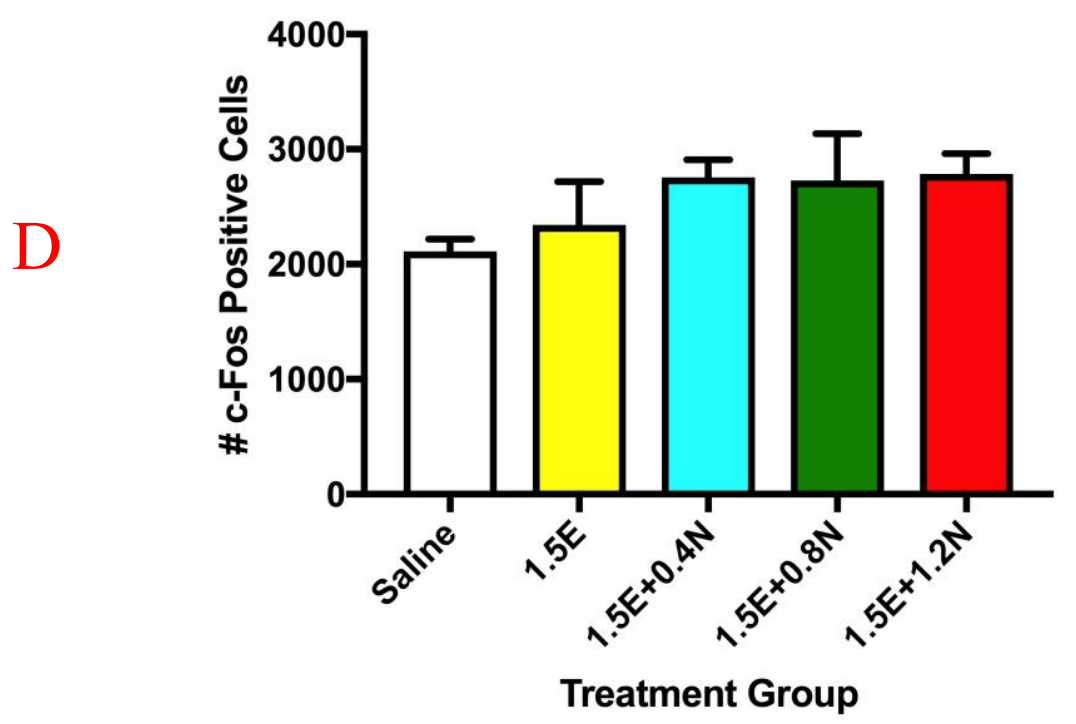

Co-administration of 0.4 and $0.8 \mathrm{mg} / \mathrm{kg}$ nicotine dose-dependently enhanced c-Fos immunoreactivity in the ventral tegmental area (VTA) compared to the ethanol only group (Graph A). The VTA is a bilateral structure that was imaged and analyzed on the left and right side separately to check for any variance between the two sides, but none was found. The centrally projecting Edinger-Westphal nucleus (cpEW) (Graph B), and the lateral septum (LS) (Graph C) when compared to alcohol only treated mice also displayed an enhanced level of c-Fos activation among the 0.4 and $0.8 \mathrm{mg} / \mathrm{kg}$ nicotine 
groups. The $0.4 \mathrm{~N}$ and $0.8 \mathrm{~N}$ groups seem to provide the most dynamic activation among the treatment groups, leading to the possibility of using these groups for further studies of stress and reward. In contrast, co-administration of $1.2 \mathrm{mg} / \mathrm{kg}$ resulted in a $33 \%$ decline in c-Fos labeled cells within the VTA, no change in LS activation, and comparable activation to $0.4 \mathrm{mg} / \mathrm{kg}$ nicotine in the cpEW. These high levels of nicotine may be overstimulating the cells in each region, but without knowing which cells in particular are activating (i.e neurons vs glia) it is difficult to conclude anything from these decreased levels of activation. Chronic intermittent nicotine exposure is altering the $\mathrm{c}$-Fos response to alcohol in a dose-dependent and brain region-selective fashion, but these results only indicate general cellular activation within the specified regions. To look in more detail at the types of cellular activation taking place in these regions we would need to label for cell types and receptors that interact with alcohol and nicotine specifically. The nearly uniform, maximal response observed following $0.8 \mathrm{mg} / \mathrm{kg}$ nicotine across stress- and reward-associated brain regions such as the NucAcc (Graph D) is consistent with the ability of this dose to accelerate the progression towards excessive alcohol selfadministration and to potentiate the discriminative stimulus effects of alcohol in rodents. Low statistical power ( $\mathrm{n}=5-6$ mice per group) in this study compared with other studies using higher populations of mice (8-12 mice per group) can contribute to the lack of significant changes of activation between groups.

\section{Conclusions}

Addiction research has come a long way in recent years, with new methods and approaches being developed constantly. As we gain a basic understanding of the neurobiology of addiction, new questions arise at every turn. Polysubstance abuse, particularly the interactions of alcohol and nicotine have major gaps that must be 
addressed by the scientific community. There is an obvious social concern with rates of comorbidity as high as $92 \%$ in people with alcohol use disorders (AUDs) (Gold, 1998). The main pharmacotherapeutic strategies do not reflect the way in which drugs are used in our society today, but focus on reducing either alcohol or nicotine use separately. None of these individual pharmacological options have proven to have the wide success that in needed to have any significant impact. People use drugs of abuse for a large variety of reasons and therapies should have a similar kind of a approach to essentially tailor to individual needs. The current research has aimed to model a chronic user of both alcohol and nicotine. Because of this, subpopulations of drug users at different stages of addiction may have an entirely different set of effects, as would be expected. Although recognizing the way in which a chronic user is functions can help identifying the discrete 'neuronal ensembles' of activation to seek out and observe in the future. The types of activation found in this study has shown a wide variety of regions that are incorporated when both alcohol and nicotine are present, but this could lead to a specific narrative that can be linked together. In addition to using knowledge of the reward system recent work has incorporated the stress system to aid in piecing together the mechanisms in facilitating the escalation of alcohol drinking with chronic nicotine users (Leão et al., 2015). With more researchers asking new questions and conducting alternative methods to studying addiction the sense of breaking through the barriers of past approaches and finding useful therapeutic treatments is that much closer to realization. 


\section{Bibliography}

Balogh, S. A., Owens, J. C., Butt, C. M., Wehner, J. M., \& Collins, A. C. (2002). Animal Models as a Tool for Studying Mechanisms of Co-Abuse of Alcohol and Tobacco. http://doi.org/10.1097/01.ALC.0000040847.98115.6D

Barrett, S. P., Tichauer, M., Leyton, M., \& Pihl, R. O. (2006). Nicotine increases alcohol self-administration in non-dependent male smokers. Drug and Alcohol Dependence. http://doi.org/10.1016/j.drugalcdep.2005.06.009

Bito-Onon, J. J., Simms, J. A., Chatterjee, S., Holgate, J., \& Bartlett, S. E. (2011). Varenicline, a partial agonist at neuronal nicotinic acetylcholine receptors, reduces nicotine-induced increases in 20\% ethanol operant self-administration in SpragueDawley rats. Addiction Biology. http://doi.org/10.1111/j.1369-1600.2010.00309.x

CDC. (2013). Tobacco Use Among Adults with Mental Illness and Substance Use Disorders. Retrieved from https://www.cdc.gov/tobacco/disparities/mental-illnesssubstance-use/index.htm

Dani, J. A., \& Harris, R. A. (2005). Nicotine addiction and comorbidity with alcohol abuse and mental illness. NATURE NEUROSCIENCE, 8(11). http://doi.org/10.1038/nn1580

Davies, M. (2003). The role of GABAA receptors in mediating the effects of alcohol in the central nervous system. Journal of Psychiatry \& Neuroscience : JPN, 28(4), 263-74. http://doi.org/PMC165791

Doyon, W. M., Dong, Y., Ostroumov, A., Thomas, A. M., Zhang, T. A., \& Dani, J. A. (2013). Nicotine decreases ethanol-induced dopamine signaling and increases selfadministration via stress hormones. Neuron.

http://doi.org/10.1016/j.neuron.2013.06.006

Falk, D. E., Yi, H. Y., \& Hiller-Sturmhöfel, S. (2006). An epidemiologic analysis of cooccurring alcohol and tobacco use and disorders: Findings from the National 
Epidemiologic Survey on Alcohol and Related Conditions. Alcohol Research and Health, 29(3), 162-171.

Ford, M. M., Fretwell, A. M., Nickel, J. D., Mark, G. P., Strong, M. N., Yoneyama, N., \& Finn, D. A. (2009). The influence of mecamylamine on ethanol and sucrose selfadministration. Neuropharmacology. http://doi.org/10.1016/j.neuropharm.2009.05.012

Ford, M. M., McCracken, A. D., Davis, N. L., Ryabinin, A. E., \& Grant, K. A. (2012). Discrimination of ethanol-nicotine drug mixtures in mice: Dual interactive mechanisms of overshadowing and potentiation. Psychopharmacology. http://doi.org/10.1007/s00213-012-2781-2

Gold, N. S. M. M. \& M. S. (1998). Cigarette Smoking and Alcohol Addiction. Journal of Addictive Diseases, 887(September). http://doi.org/10.1300/J069v17n01

Hurley, L. L., Taylor, R. E., \& Tizabi, Y. (2012). Positive and negative effects of alcohol and nicotine and their interactions: A mechanistic review. Neurotoxicity Research. http://doi.org/10.1007/s12640-011-9275-6

ImageJ. (n.d.). No Title. Retrieved from https://imagej.nih.gov/ij/

Kalivas, P. W., Szumlinski, K. K., Abernathy, K. E., Oleson, E. B., Klugmann, M., Lominac, K. D., ... Kalivas, P. W. (2006). Homer Isoforms Differentially Regulate Cocaine-Induced Neuroplasticity. Neuropsychopharmacology, 31(4), 768-777. http://doi.org/10.1038/sj.npp.1300890

Koob, G. F. (1997). Drug Abuse: Hedonic Homeostatic Dysregulation. Science, 278(5335), 52-58. http://doi.org/10.1126/science.278.5335.52

Koob, G. F., Buck, C. L., Cohen, A., Edwards, S., Park, P. E., Schlosburg, J. E., ... George, O. (2014). Addiction as a stress surfeit disorder. Neuropharmacology, 76(PART B), 370-382. http://doi.org/10.1016/j.neuropharm.2013.05.024

Koob, G. F., \& Volkow, N. D. (2010). Neurocircuitry of addiction. Neuropsychopharmacology: Official Publication of the American College of Neuropsychopharmacology, 35(1), 217-238. http://doi.org/10.1038/npp.2009.110 
Lê, A. D., Lo, S., Harding, S., Juzytsch, W., Marinelli, P. W., \& Funk, D. (2010).

Coadministration of intravenous nicotine and oral alcohol in rats. Psychopharmacology. http://doi.org/10.1007/s00213-009-1746-6

Leão, R. M., Cruz, F. C., Leandro, X., Vendruscolo, F., De Guglielmo, G., Logrip, M. L., ... George, X. O. (2015). Behavioral/Cognitive Chronic Nicotine Activates Stress/Reward-Related Brain Regions and Facilitates the Transition to Compulsive Alcohol Drinking. http://doi.org/10.1523/JNEUROSCI.3302-14.2015

Matta, S. G., Valentine, J. D., \& Sharp, B. M. (1997). Nicotinic activation of CRH neurons in extrahypothalamic regions of the rat brain. Endocrine, 7(2), 245-53. http://doi.org/10.1007/BF02778147

OHSU. (n.d.). Andrey Ryabinin. Retrieved from http://www.ohsu.edu/xd/education/schools/school-of-medicine/academicprograms/graduate-studies/faculty/grad-studies-faculty.cfm?facultyid=315

Ryabinin, A. E., Criado, J. R., Henriksen, S. J., Bloom, F. E., \& Wilson, M. C. (1997). Differential sensitivity of c-Fos expression in hippocampus and other brain regions to moderate and low doses of alcohol. Molecular Psychiatry, 2(1), 32-43. Retrieved from http://www.ncbi.nlm.nih.gov/pubmed/9154216

Sng, J. C. G., Taniura, H., \& Yoneda, Y. (2004). A tale of early response genes. Biological \& Pharmaceutical Bulletin, 27(5), 606-12. http://doi.org/10.1248/bpb.27.606

Vilpoux, C., Warnault, V., Pierrefiche, O., Daoust, M., \& Naassila, M. (2009). Ethanolsensitive brain regions in rat and mouse: A cartographic review, using immediate early gene expression. Alcoholism: Clinical and Experimental Research. http://doi.org/10.1111/j.1530-0277.2009.00916.x

Walker, J. J., Spiga, F., Waite, E., Zhao, Z., Kershaw, Y., Terry, J. R., \& Lightman, S. L. (2012). The Origin of Glucocorticoid Hormone Oscillations. PLoS Biology, 10(6), e1001341. http://doi.org/10.1371/journal.pbio.1001341 
End. 